\title{
Pathway and Energetics of the Thermally-Induced Structural Changes in Microemulsions
}

\author{
Yadong He \\ Department of Mechanical Engineering, Virginia Tech \\ 460 Old Turner St., Blacksburg, VA 24061, USA \\ Email: yadongh@vt.edu \\ Ying Liu \\ Department of Mechanical Engineering, Virginia Tech \\ 460 Old Turner St., Blacksburg, VA 24061, USA \\ Email: yliu4@vt.edu \\ Bao Yang \\ Department of Mechanical Engineering, University of Maryland, College Park \\ 4164D Glenn L. Martin Hall, University of Maryland, College Park, MD 20742 \\ Email: baoyang@umd.edu

\section{Rui Qiao ${ }^{1}$} \\ Department of Mechanical Engineering, Virginia Tech \\ 460 Old Turner St., Blacksburg, VA 24061, USA \\ Email: ruiqiao@vt.edu
}

\begin{abstract}
Microemulsions are thermodynamically stable dispersions of nano-droplets stabilized by surfactants. In water-in-oil microemulsions, the nano-droplets exist in the form of swollen reverse micelles. Recent experiments demonstrated very high critical heat fluxes (CHF) in boiling of water-in-oil microemulsions, which makes these microemulsions ideal heat transfer fluids for high-heat flux thermal management applications. While the nanoscale structure of the microemulsions is inevitably changed during their boiling, little is known about such change and its energetics, which are critical for understanding the microemulsion boiling. Here we report a study of the pathway and energetics of thermally-induced structural change in water-in-oil microemulsions using microsecond-long molecular dynamics simulations. We show that, for microemulsions that are thermodynamically stable at room temperature, upon increasing their temperature to similar to that found in boiling experiments, their spherical reverse micelles transition to cylindrical wormlike reverse micelles through merging and splitting of individual reverse micelles. These structural transitions are accompanied by a gradual release of water molecules from the reverse micelles. These transitions are endothermic with the heat absorption dominated by the release of water molecules from the water core of the reverse micelles. The release rate of water molecules from the interior of reverse micelles, hence the heat absorption rate, slows down as the structural transition proceeds because the remaining water molecules in the interior bind more closely to the polar moieties of the surfactants.
\end{abstract}

Keywords: microemulsions, nanofluids, thermal transport, phase change, self-assembly, molecular dynamics

\footnotetext{
${ }^{1}$ To whom correspondence should be addressed.
} 


\section{INTRODUCTION}

Stable mixture of polar and apolar liquids is difficult to achieve due to the segregation tendency of these liquids. However, when suitable type and amount of surfactants are introduced, thermodynamically stable dispersions of polar liquids in apolar liquids (or vice versa) can be obtained and these dispersions are termed microemulsions. Two common types of microemulsions are water-inoil microemulsions and oil-in-water microemulsions [1]. Here "water" refers to a polar phase and "oil" refers to an apolar organic phase. The dispersed phase often exists as nanostructured entities stabilized by surfactant molecules. These entities, with characteristic dimensions as small as a few angstroms are termed swollen reverse micelles (RM) in water-in-oil microemulsions or swollen micelles in oil-in-water microemulsions [1]. Because of their unique properties such as the nanoscale size of their internal structures, microemulsions have a wide range of applications including drug delivery, chemical synthesis, and enhanced oil recovery [2-4].

While microemulsions have been used extensively in industrial applications since their discovery, they have rarely been used in heat transfer applications until recently [5-8]. In particular, one of us has developed water-in-polyalphaolefin (PAO) microemulsions as heat transfer fluids for high heatflux cooling [5-7]. At low water loading and room temperature, this microemulsion features spherical RMs, whose radii vary from 1.3 to $9.6 \mathrm{~nm}$ when the water concentration in the microemulsion increases from 1.8 to $4.5 \%$. The viscosity and thermal conductivity of this microemulsion is only slightly higher $(<20 \%)$ than that of the pure PAO liquids. Using this microemulsion as heat transfer fluids, nucleate boiling was observed in the pool boiling experiments and critical heat flux (CHF) as high as $500 \mathrm{~W} / \mathrm{cm}^{2}$ was achieved without modifying the microstructure of the heater surfaces [7]. The water vapor released during boiling, once condensed, self-assembled spontaneously with the surfactant molecules in the liquids to form RMs, which makes microemulsion suitable for closed-loop operations in thermal management system. These results suggest that microemulsions may offer a new method for achieving 
superior CHF in practical engineering applications. This method is potentially more robust compared to the classical method of introducing microstructures to the heater surfaces. This is because, the microstructure of heater surfaces is often inevitably modified during operation (e.g., by fouling), which compromises the CHF in long-term operations and/or in aggressive working environments. For example, it is known that the enhancement of CHF observed for nanofluids is highly variable and often deteriorates over time [9].

The fundamental understanding of the boiling of microemulsions is limited at present. This, along with the fact that numerous microemulsions can be engineered by tuning the type and loading of the polar/apolar fluids and the surfactant molecules, makes it difficult to optimize microemulsions for achieving better boiling performance. To gain insight into the boiling of microemulsions, it is essential to clarify the formation, growth, and departure of bubbles, and how these bubble dynamics are coupled with the fluid flow and heat transfer during boiling. While these problems have been clarified to a large extent for boiling of pure liquids in prior research, applying the insight from those prior studies to the boiling of microemulsions is not straightforward due to the emergence of new issues. Some of the most important new issues are how the structure of the RMs changes in response to elevated temperature, how polar molecules are released during boiling of microemulsions, and how heat is absorbed during this process. These issues are unique to the boiling of microemulsion, and clarifying them is a necessary step toward understanding the microemulsion boiling.

While the phenomena of polar molecules being released from RMs during boiling of microemulsions and the associated heat absorption are evident in experiments (e.g., formation of visible bubbles and effective heat removal from heater surfaces), little is known about the nature of these processes. Conceivably, the internal nanostructures of the microemulsions are changed during boiling, but it is not clear what pathways the structure change follows and how they affects the water release 
and heat absorption. Such a limited understanding, despite decades of study of microemulsions, is due to the fact that the phase and structural change of microemulsions under conditions similar to that in boiling of microemulsions was rarely studied in the past. Specifically, prior studies focused on phase change at moderate temperature (typically $<60^{\circ} \mathrm{C}$, i.e., much lower than the boiling temperature of water) while temperature higher than $100^{\circ} \mathrm{C}$ is often found in boiling of microemulsions [7]. Prior studies of the phase change in microemulsions were mostly performed under quasi-equilibrium conditions (i.e., the temperature was changed very slowly), but the temperature of microemulsions changes greatly over millisecond time scales as they move past the heater surface.

In the present work, we investigate the evolution of the internal nanostructure of microemulsions at high temperature and the associated energy changes. Specifically, we examine how water molecules are released from water-in-oil microemulsions when the temperature of the microemulsion is raised to temperature close to the boiling temperature of bulk water using microsecond-long molecular dynamics (MD) simulations. MD simulations have been used successfully to study self-assembly of micelles [10-23], thermal transport, and phase changes [24, 25], and are thus useful to study the thermally-induced changes of microemulsions. Here, the release of water molecules and the associate heat absorption are quantified, and the molecular details of the structural change of the microemulsions during water release are provided. The rest of the paper is organized as follows. Section 2 presents the molecular models and methods for our MD simulations. Section 3 presents the structural changes and associated energy change of the microemulsions at high temperature. Finally, conclusions are drawn in Section 4.

\section{SIMULATION MODELS AND METHODS}

We use MD simulations to study the thermally-induced structural change of water-in-oil microemulsions. Figure 1 shows a sketch of the simulation system. The system includes two water- 
swollen RMs stabilized by sodium bis(2-ethylhexyl)sulfosuccinate (AOT) and the oil phase of the microemulsion is taken as n-octane fluids. Each RM consists of 42 AOT molecules and 210 water molecules (note that each AOT molecule contains one $\mathrm{Na}^{+}$ion). This gives a water-to-surfactant ratio $\left(W_{0}=\left[\mathrm{H}_{2} \mathrm{O}\right] /[\mathrm{AOT}]\right)$ of five, which is common for water-in-oil microemulsions. There are $6000 \mathrm{n}$ octane inside the system to ensure a high mass fraction of the n-octane liquids. The microemulsion is placed on top of a solid wall to form a film of $\sim 11 \mathrm{~nm}$ in thickness at $300 \mathrm{~K}$. The wall is modeled as a square lattice of carbon atoms. The system contains a total of 175348 atoms. A free space is introduced above the microemulsion film to accommodate the water molecules released from the RMs during the simulations. The simulation box measures $18.8 \times 9.4 \mathrm{~nm}^{2}$ in the $x y$-plane and $20 \mathrm{~nm}$ in the z-direction. Periodical boundary conditions are applied in all three directions.

All-atom models are adopted for the AOT, water, and n-octane molecules. The SPC/E model is used for the water molecules. The force fields for AOT molecules are the same as those used by Abel et al. [10, 26], which were derived from the CHARMM family of force fields [27]. The force fields for the $n$ octane molecules are derived from the CHARMM36 all-atom parameters for lipids [27]. Previous MD simulations indicated that correct water-AOT self-assembly behavior can be captured using these force fields $[10,17,20]$. Simulations of $n$-octane fluids at $1 \mathrm{~atm}$ and $298 \mathrm{~K}$ showed that key properties such as density and thermal conductivity are reproduced very well using the force fields for n-octane fluids. The wall atoms are modeled as frozen neutral carbon atoms, and their Lennard-Jones parameters are taken from [28].

To generate the simulation system, we first build two RMs using water and AOT molecules. Specifically, we use the PACKMOL code [29] to pack the desired number of water molecules into a spherical space and the AOT molecules (including the $\mathrm{Na}^{+}$ion) into a spherical shell surrounding the water core. These RMs are then positioned at a distance several nanometers above the solid wall, and the desired number of n-octane molecules are packed into the simulation box to make a film of 
microemulsion over the wall. The MD system thus generated is relaxed through energy minimization, and then equilibrated for $20 \mathrm{~ns}$ with the position of the RMs restrained. This is followed by a $4 \mathrm{~ns}$ equilibration of the whole system at $300 \mathrm{~K}$. The equilibrated system is then taken as the starting configuration of the subsequent simulations. To study the thermally-induced structural change in microemulsions, we performed two sets of simulations. In the first set of simulations, the microemulsion generated above is equilibrated at $300 \mathrm{~K}$ for $\sim 0.8 \mu$ s to assess the stability of the RMs. The system studied in this simulation is hereafter referred to as the "reference system". In the second set of simulations, the system temperature is increased by $20 \mathrm{~K}$ every $4 \mathrm{~ns}$ until the temperature reaches 370 K. After that, the temperature of the microemulsion is maintained at $370 \mathrm{~K}$ and a production run of $\sim 0.8$ $\mu \mathrm{s}$ is performed. During the production run, water molecules are released from the RMs and enter the free space above the microemulsion film. Since the released water molecules left the system in the form of vapor bubbles in real boiling experiments, the water molecules entering into the free space above the microemulsion film is removed from the system every $20 \mathrm{~ns}$. The system studied in this simulation is termed as the "heated system". We note that the MD simulations of both the reference system and the heat system last 10-100 times longer than those reported in the literature (typically abo1ut a few to tens of nanoseconds [10-18]). These microsecond-long simulations are essential for assessing the stability of RMs at the room temperature and elevated temperature and for exploring the pathways for the thermally-induced structural changes of RMs.

We use the GROMACS code [30-32] to perform the above MD simulations. In these simulations, the temperature of the microemulsion is maintained using the Nosé-Hoover thermostat with a time constant of $0.4 \mathrm{ps}$. The Lennard-Jones interactions are calculated by direct summation at distance up to $0.8 \mathrm{~nm}$ and switched off smoothly to zero at distance of $1.1 \mathrm{~nm}$. The electrostatic interactions are computed using the Particle-Mesh Ewald (PME) method with a real-space cutoff length of $1.2 \mathrm{~nm}$, and a FFT spacing of $0.14 \mathrm{~nm}$. All bonds are constrained using the LINCS algorithm [33], which enables a time 
step of 2 fs to be used in the simulations. The trajectory of the system is saved every 50 ps for analysis.

\section{RESULTS AND DISCUSSION}

\subsection{Stable microemulsions at $300 \mathrm{~K}$}

To assess whether our MD models enable simulation of stable microemulsions at room temperature, we first examine the two RMs in the reference system, in which microemulsion is maintained at $300 \mathrm{~K}$. Visualization of the trajectory indicates that the two RMs remain intact during the entire simulation. To quantitatively characterize these RMs, we compute their eccentricity. Following $[14,20,34]$, we define the eccentricity $\epsilon$ as,

$\epsilon=1-I_{\min } / I_{\text {avg }}$

where $I_{\min }$ and $I_{\text {avg }}$ are the minimal and average value of the RM's principal moment of inertia, respectively. An eccentricity of $\epsilon=0$ corresponds to a perfectly spherical RM. The principal moment of inertia is determined by calculating the eigenvalues of the inertia tensor $\boldsymbol{I}$ of the RM, which is assembled as

$\boldsymbol{I}=\left[\begin{array}{lll}I_{x x} & I_{x y} & I_{x z} \\ I_{y x} & I_{y y} & I_{y z} \\ I_{z x} & I_{z y} & I_{z z}\end{array}\right]$

where $I_{x x}, I_{y y}, I_{z z}$ are the moment of inertia about the $x-, y-$, and $z$ - axis, respectively. $I_{x y}$ is defined as the $x y$ product of inertia, and other moment of inertia such as $I_{y x}$ are defined in similar ways. $I_{x x}$ and $I_{x y}$ are evaluated using

$I_{x x}=\sum_{i=1}^{N}\left(y_{i}^{2}+z_{i}^{2}\right) m_{i}$

$I_{x y}=-\sum_{i=1}^{N} x_{i} y_{i} m_{i}$

where $\mathrm{N}$ is the number of atoms in the $\mathrm{RM}, x_{i}, y_{i}, z_{i}, m_{i}$ are the $x, y, z$ coordinates and the mass of atom $i$. Other moments of inertia such as $I_{x y}$ and $I_{y y}$ are computed using similar equations. 
It is worth noting that, when assembling the inertia tensor, it is important to take into account the periodical boundary condition because an RM may cut across the boundary of the simulation box. Figure 2 shows that, while the RMs are spherical on average, they can become elongated during certain time period and their eccentricity fluctuates moderately. From these results, the eccentricity of the RMs is found to be $\epsilon=0.25 \pm 0.07$. The magnitude of the fluctuation of the RMs' eccentricity is similar to that found in recent microsecond-long MD simulations of AOT RMs in iso-octane formed by spontaneous self-assembly [20]. These results confirm that packing water molecules and AOT molecules into spherical RMs (termed as the "pre-assembled approach" in the literature) can both significantly accelerate the formation of RMs and enable simulation of stable microemulsions. It is worth noting that because of the continuous fluctuation of the reverse micelle's shape, water molecules may briefly become not fully shielded by the hydrophilic head groups (see Figure 2). This phenomenon is often observed in molecular simulation of reverse micelles (see, e.g., Figure 1a of Ref. [10]), and it is not an indication of the instability of the reverse micelle. In addition, the dynamic exchange of water molecules between core fluids of reverse micelles and/or bulk oil phase is often observed in thermodynamically stable microemulsions [35]. In our simulations at 300K, although water molecules stay mostly inside the microemulsions, a few of them occasionally escape from one reverse micelle into the oil. Nevertheless, the escaped water molecules always migrate back to its original reverse micelle or the other reverse micelle nearby within nanosecond time scale. Finally, although the two RMs in the microemulsions can interact with each other, they are both stable at room temperature and do not merge with each other over the time scale explored here. 
To help understand the change of the nanoscale structure of microemulsions (or more specifically the structure of the RMs within the microemulsion) induced by elevated temperature, we further characterize the molecular structure of the RMs. Figure 3 shows the density profiles of the water molecules, $\mathrm{Na}^{+}$ions, and AOT head/tail across the spherical RMs. The water density profile suggests that the water core of the RM has a radius of $\sim 1.0 \mathrm{~nm}$. The fact that the water density is close to $1 \mathrm{~g} / \mathrm{cc}$ within $\sim 0.7 \mathrm{~nm}$ from the center of RM suggests that the water molecules in the inner part of the water core are bulk-like. The facts that water density decreases as one moves from the interior of water core toward the hydrophobic tail of the AOT molecules and reaches nearly zero at position beyond the position of the hydrophilic heads of the AOT molecules suggest that water molecules are packed tightly inside the cage formed by the AOT's hydrophilic head groups. Figure 2 also shows that the $\mathrm{Na}^{+}$ions are localized near AOT molecules' hydrophilic head groups rather than in the interior of the water core. This is consistent with previous MD simulations of RMs featuring AOT surfactants [10], and it is caused by the fact that the charge-charge interaction between $\mathrm{Na}^{+}$ions and AOT's head group is stronger than the charge-dipole interactions between $\mathrm{Na}^{+}$ions and water molecules.

\subsection{Structural evolution of microemulsions at $370 \mathrm{~K}$}

Figure 4 shows the snapshots of the two RMs (labeled as RM1 and RM2) in the microemulsion film positioned on top of the solid wall after its temperature was increased from $300 \mathrm{~K}$ to $370 \mathrm{~K}$. While the two RMs remain intact during a $0.8 \mu \mathrm{s}$ simulation at $300 \mathrm{~K}$, they go through significant structural changes at high temperature. At $370 \mathrm{~K}$, their shape fluctuation is much stronger than that at $300 \mathrm{~K}$, and both RMs deviate from the nearly spherical shape and become adsorbed on the solid wall by $t \approx 20 \mathrm{~ns}$. Nevertheless, the adsorption does not seem to 
greatly change the organization of the various species within the RM, e.g., most water molecules are still packed inside the space enclosed by the hydrophilic heads of the AOT molecules. This is likely due to the fact that the solid wall made of carbon atoms is hydrophobic in nature.

The strong shape fluctuations of the RMs lead to the splitting of RM2 at $t \approx 120 \mathrm{~ns}$. While one of the daughter RMs (labeled as RM2a) remains spherical for another more than $400 \mathrm{ns,}$ the other one (RM2b) soon fuses with RM1 and forms a wormlike micelle (W1) at $t \approx 280$ ns. The wormlike micelle thus formed, however, is not stable and splits into one small RM (W1a) and one wormlike micelle (W1b) by $t \approx 400 \mathrm{~ns}$, which later merge again by $t \approx 480 \mathrm{~ns}$ forming a new cylindrical wormlike micelle (W2). The remaining small spherical RM (RM2a) then fuses with the cylindrical wormlike micelle (W2) by $t \approx 560 \mathrm{~ns}$, and the system contains only one single large cylindrical wormlike micelle (W3) about $18 \mathrm{~nm}$ long at $t=800 \mathrm{~ns}$.

The strong shape fluctuation and structure transition of the RMs shown in Figure 4 is accompanied by a change of the water content of the RMs. As shown in Figure $4 \mathrm{~b}$, some of the water molecules (marked by an arrow) are not well "protected" by the hydrophilic heads of the AOT molecules and become partly exposed to the n-octane oil, which facilitates the release of water molecules from the RMs. Visualization of the trajectories indicated that, once water molecules diffuse into the n-octane oil, they may either diffuse back into the RM or diffuse into the free space above the microemulsion film. Figure 5 shows that the number of water molecules inside the RMs changes very little during the first 100 ns, during which neither RMs split nor fuse with each other. At $t>100$ ns, the number of water molecules inside the RMs 
gradually decreases as the RMs go through structural transition. Interestingly, we observe that the water loss rate decreases as time increases, i.e., it becomes increasingly more difficult for water molecules to diffuse out of the RMs. To understand this, we analyze the microenvironment of the water molecules inside the RMs as they go through structural transitions. Specifically, we compute the density distribution of various species around a water molecule inside the RMs. Figure 6 compares these density profiles during $t=0-20$ ns and during $t=780-800$ ns. It shows that, at $\mathrm{t}=780-800 \mathrm{~ns}$, a water molecule within an $\mathrm{RM}$ is surrounded by more $\mathrm{Na}^{+}$ ions and the hydrophilic heads/hydrophobic tails of the AOT surfactant and by less water molecules compared to that during $t=0-20 \mathrm{~ns}$. This partly reflects the fact that more water molecules are distributed near the AOT's head group rather than in the interior of the water core during $t=780-800$ ns (i.e., there are less free water molecules but more bonded water molecules at large time, which is consistent with the larger surfactant/water ratio). It also suggests that water molecules in contact with the hydrophilic moieties of the AOT molecules are better coordinated with these moieties at large times. Since the electrostatic charge-dipole interactions between water- $\mathrm{Na}^{+}$ion and water-hydrophilic heads are stronger than the electrostatic dipole-dipole interactions between water molecules, it follows that water molecules in RMs at large time ( $t=780-800 \mathrm{~ns}$ ) are in energetically more favorable states compared to that at the short time $(t=0-20 \mathrm{~ns})$. Consequently, it is more difficult for water molecules to diffuse out of the RMs at large times.

The above results suggest that the microemulsion studied here, while stable at $300 \mathrm{~K}$, go through complex internal structural changes as its temperature is elevated to $370 \mathrm{~K}$. During these changes, RMs can split into smaller RMs and also merge to form cylindrical wormlike 
micelles, which can again merge with other RMs and split into smaller RMs. Limited by computational resources, we cannot explore the structural evolution of the wormlike micelle at even larger time scale and the possible interactions between multiple wormlike micelles. However, conceivably, wormlike micelles can also merge with each other to form extended networks, which may be disrupted by the fluid flows generated during boiling. Based on these results, we can expect that, during the boiling of microemulsions, there should exist a spectrum of molecular and mesoscale self-assembled structures inside the microemulsions that are absent at room temperatures. These structures can potentially modify the thermophysical properties of the microemulsions greatly. For example, the formation of longer or even connected networks of cylindrical wormlike micelles should increase the viscosity of the fluids locally, which directly impact the fluid transport and thus the bubble dynamics.

\subsection{Energetics of the structural evolution of microemulsions at $370 \mathrm{~K}$}

The structural change of the RMs within microemulsion is accompanied by a change of the total energy of the microemulsion system. Figure 7 shows that, while the temperature of the microemulsion is maintained at $370 \mathrm{~K}$, its total energy increases as a function of time, i.e., heat is absorbed by the microemulsions during its structural transition (note that heat absorption is realized through the application of thermostat in our MD simulations). Such a heat absorption is akin to the heat absorption during phase change of pure fluids, and it is consistent with the heat absorption observed during the boiling of microemulsions. It is desirable to compare the heat adsorption computed here with experimental measurements. However, there is not yet any available data for the heat adsorption associated with thermal decomposition of microemulsions at elevated temperature (especially close to the boiling 
temperature of the core fluids) due to the short history of research on microemulsion boiling. Hence we now proceed directly to the analysis of the energetics associated with the thermal decomposition of microemulsions.

The heat absorption shown in Figure 7 is mainly caused by the transfer of water molecules from the interior of RMs into the n-octane oil and the free space. To gain insight into the energetics of such transfer, we compute the interaction energy of a single water molecule with its surrounding molecules. While it is straightforward to compute the interaction energy due to the short-ranged van der Waals interactions (modeled using the Lennard-Jones potential in our simulations), it is difficult to compute the contribution of electrostatic interactions to the total interaction energy of one water molecules with other molecules inside the system. This is because, when the long-ranged electrostatic interactions are computed using the PME method, the electrostatic interactions are split into a short-ranged real-space part and a long-ranged kspace part. The split is controlled by the real-space cutoff $r_{r, \text { cut }}$ : as $r_{r, c u t}$ increases, the realspace (k-space) part of the interactions increases (decreases). One can determine the realspace part of the electrostatic interaction between a charged atom and its surrounding atoms by direct summation. However, the k-space part of the electrostatic interactions is computed for all charged atoms in the system together and thus is difficult to be divided among different molecules. Here, we note that the total energy due to the electrostatic interactions of a water molecule (a dipole) with its surrounding molecules is dominated by its interactions with the molecules very close to it, and the energy of these interactions are included in the real-space part of electrostatic interaction to a large extent, especially when $r_{r, c u t}$ is large. Table 1 shows 
the interaction energy of a water molecule with its surrounding medium when it is in different environments (the results are qualitatively similar for the two different $r_{r, c u t}$ used).

The interaction energy is close to zero when the water molecule is in the free space (i.e., gas phase) since the separation between water molecules is large. When the water molecule is inside the $\mathrm{n}$-octane liquids, the interaction energy is small $(\sim-7.5 \mathrm{~kJ} / \mathrm{mol})$ because it is dominated by the weak van der Waals interactions between water and the apolar n-octane oil. However, when the water molecule is enclosed inside an RM, the interaction energy is very large $(\sim-70 \mathrm{~kJ} / \mathrm{mol})$ due to the strong electrostatic interactions between the water molecules and the hydrophilic head/Na+ ions of the AOT surfactants. Based on the data shown in Table 1, when a water molecule is released from an RM into the n-octane oil, heat absorption on the order of $\sim 60-70 \mathrm{~kJ} / \mathrm{mol}$ is expected; a further heat absorption of $\sim 7 \mathrm{~kJ} / \mathrm{mol}$ is expected when the water molecule diffuses from the oil into the gas phase. Using the energy data shown in Figure 7 and the evolution of water molecules inside RMs shown in Figure 5, we find that a heat absorption of $\sim 31 \mathrm{~kJ} / \mathrm{mol}$ is needed for each water molecule released into the gas phase. This heat absorption is about $50 \%$ of that estimated above, and the difference originates from the fact that, in the above estimations, only the interactions between water molecules and its surrounding medium are considered. In reality, when water molecules are released from an $\mathrm{RM}$, the hydrophilic moieties (the hydrophilic head and the $\mathrm{Na}^{+}$ions) of the AOT surfactants become more closely packed, and the interactions between them become stronger. The latter partially offsets the increase of the system energy due to the release of water molecules into the gas phase. 


\section{CONCLUSIONS}

The evolution of the internal structure of water-in-octane microemulsions induced by elevated temperature is studied using all-atom MD simulations. The simulations reveal that RMs that are stable at room temperature show strong shape fluctuation at elevated temperatures, which facilitates the splitting of initially spherical RMs. The daughter RMs generated during such splitting can merge with each other to form cylindrical wormlike micelles. Because these different structures can transition between each other, a wide spectrum of structures including small spherical RMs and cylindrical wormlike micelles can be observed in microemulsions at elevated temperature. Some of these structures such as wormlike micelles will likely change the viscosity of the microemulsions greatly and thus affect their boiling behavior. Water molecules are released from RMs during their structural transition, and the water release rate gradually decreases as the water content in the RMs decreases due to the stronger interactions of the remaining water with the hydrophilic moieties of the surfactant molecules. The heat absorption during the structural transition of microemulsions is dominated by the energy change of the water molecules released from the interior of the RMs into the apolar n-octane oil.

\section{ACKNOWLEDGMENT}

The authors thank the CCIT office at Clemson University and the ARC at Virginia Tech for their generous allocation of computer time on the Palmetto, BlueRidge, and NewRiver clusters. R.Q. and B.Y. gratefully acknowledge the financial support from National Science Foundation (NSF) under Grant 1463932 and Grant 1336778, respectively. 


\section{REFERENCES}

[1] M.K. Sharma, D.O. Shah, Introduction to macro- and microemulsions, in: Macro- and Microemulsion: Theory and Applications, Vol. 272, American Chemical Society, Washington, D.C., 1985, pp. 1-18.

[2] M.P. Pileni, Reverse micelles as microreactors, J. Phys. Chem. 97 (1993) 6961-6973.

[3] R. Trivedi, U.B. Kompella, Nanomicellar formulations for sustained drug delivery: strategies and underlying principles, Nanomedicine 5 (2010) 485-505.

[4] M.K. Sharma, D.O. Shah, Macro- and microemulsions in enhanced oil recovery, in: Macroand Microemulsion: Theory and Applications, Vol. 272, American Chemical Society, Washington, D.C., 1985, pp. 149-172.

[5] J.J. Xu, B. Hammouda, F.Y. Cao, B. Yang, Experimental study of thermophysical properties and nanostructure of self-assembled water/polyalphaolefin nanoemulsion fluids, Adv. Mech. Eng. 7 (2015) 1-8.

[6] J.J. Xu, B. Yang, B. Hammouda, Thermal conductivity and viscosity of self-assembled alcohol/polyalphaolefin nanoemulsion fluids, Nanoscale Res. Lett. 6 (2011) 1-6.

[7] J.J. Xu, B. Yang, B. Hammouda, Thermophysical properties and pool boiling characteristics of water-in-polyalphaolefin nanoemulsion fluids, J. Heat Transf.-Trans. ASME 135 (2013) 321-325.

[8] M. Chiesa, J. Garg, Y.T. Kang, G. Chen, Thermal conductivity and viscosity of water-in-oil nanoemulsions, Colloids Surf., A 326 (2008) 67-72.

[9] H. Kim, Enhancement of critical heat flux in nucleate boiling of nanofluids: a state-of-art review, Nanoscale Res. Lett. 6 (2011) 1-18.

[10] S. Abel, F. Sterpone, S. Bandyopadhyay, M. Marchi, Molecular modeling and simulations of AOT-water reverse micelles in isooctane: structural and dynamic properties, J. Phys. Chem. B 108 (2004) 19458-19466.

[11] S. Abel, M. Waks, M. Marchi, W. Urbach, Effect of surfactant conformation on the structures of small size nonionic reverse micelles: a molecular dynamics simulation study, Langmuir 22 (2006) 9112-9120.

[12] S. Abel, M. Waks, W. Urbach, M. Marchi, Structure, stability, and hydration of a polypeptide in AOT reverse micelles, J. Am. Chem. Soc. 128 (2006) 382-383.

[13] S. Abel, J. Attia, S. Remita, M. Marchi, W. Urbach, M. Goldmann, Atomistic simulations of spontaneous formation and structural properties of linoleic acid micelles in water, Chem. Phys. Lett. 481 (2009) 124-129. 
[14] J. Chowdhary, B.M. Ladanyi, Molecular dynamics simulation of aerosol-OT reverse micelles, J. Phys. Chem. B 113 (2009) 15029-15039.

[15] S. Abel, M. Waks, M. Marchi, Molecular dynamics simulations of cytochrome c unfolding in AOT reverse micelles: the first steps, Eur. Phys. J. E 32 (2010) 399-409.

[16] S. Abel, F.-Y. Dupradeau, E.P. Raman, A.D. MacKerell, M. Marchi, Molecular simulations of dodecyl- $\beta$-maltoside micelles in water: influence of the headgroup conformation and force field parameters, J. Phys. Chem. B 115 (2011) 487-499.

[17] S. Abel, F.-Y. Dupradeau, M. Marchi, Molecular dynamics simulations of a characteristic DPC micelle in water, J. Chem. Theory Comput. 8 (2012) 4610-4623.

[18] A.V. Martinez, L. Dominguez, E. Malolepsza, A. Moser, Z. Ziegler, J.E. Straub, Probing the structure and dynamics of confined water in AOT reverse micelles, J. Phys. Chem. B 117 (2013) 7345-7351.

[19] S. Manet, A.-S. Cuvier, C. Valotteau, G.C. Fadda, J. Perez, E. Karakas, S. Abel, N. Baccile, Structure of bolaamphiphile sophorolipid micelles characterized with SAXS, SANS, and MD simulations, J. Phys. Chem. B 119 (2015) 13113-13133.

[20] M. Marchi, S. Abel, Modeling the self-aggregation of small AOT reverse micelles from firstprinciples, J. Phys. Chem. Lett. 6 (2015) 170-174.

[21] V.R. Vasquez, B.C. Williams, O.A. Graeve, Stability and comparative analysis of AOT/water/isooctane reverse micelle system using dynamic light scattering and molecular dynamics, J. Phys. Chem. B 115 (2011) 2979-2987.

[22] A.V. Nevidimov, V.F. Razumov, Molecular dynamics simulations of AOT reverse micelles' self-assembly, Mol. Phys. 107 (2009) 2169-2180.

[23] C.D. Bruce, M.L. Berkowitz, L. Perera, M.D.E. Forbes, Molecular dynamics simulation of sodium dodecyl sulfate micelle in water: Micellar structural characteristics and counterion distribution, J. Phys. Chem. B 106 (2002) 3788-3793.

[24] Y. Zou, X. Huai, L. Lin, Molecular dynamics simulation for homogeneous nucleation of water and liquid nitrogen in explosive boiling, Appl. Therm. Eng. 30 (2010) 859-863.

[25] Y. Mao, Y. Zhang, Molecular dynamics simulation on rapid boiling of water on a hot copper plate, Appl. Therm. Eng. 62 (2014) 607-612.

[26] A. Maitra, Determination of size parameters of water-Aerosol OT-oil reverse micelles from their nuclear magnetic resonance data, J. Phys. Chem. 88 (1984) 5122-5125. 
[27] P. Bjelkmar, P. Larsson, M.A. Cuendet, B. Hess, E. Lindahl, Implementation of the CHARMM force field in GROMACS: analysis of protein stability effects from correction maps, virtual interaction sites, and water models, J. Chem. Theory Comput. 6 (2010) 459-466.

[28] G. Hummer, J.C. Rasaiah, J.P. Noworyta, Water conduction through the hydrophobic channel of a carbon nanotube, Nat. 414 (2001) 188-190.

[29] L. Martinez, R. Andrade, E.G. Birgin, J.M. Martinez, PACKMOL: a package for building initial configurations for molecular dynamics simulations, J. Comput. Chem. 30 (2009) 2157-2164.

[30] D. Van der Spoel, E. Lindahl, B. Hess, G. Groenhof, A.E. Mark, H.J.C. Berendsen, GROMACS: fast, flexible, and free, J. Comput. Chem. 26 (2005) 1701-1718.

[31] H.J.C. Berendsen, D. Vanderspoel, R. Vandrunen, GROMACS: a message-passing parallel molecular dynamics implementation, Comput. Phys. Commun. 91 (1995) 43-56.

[32] S. Pronk, S. Pall, R. Schulz, P. Larsson, P. Bjelkmar, R. Apostolov, M.R. Shirts, J.C. Smith, P.M. Kasson, D. van der Spoel, B. Hess, E. Lindahl, GROMACS 4.5: a high-throughput and highly parallel open source molecular simulation toolkit, Bioinform. 29 (2013) 845-854.

[33] B. Hess, H. Bekker, H.J.C. Berendsen, J. Fraaije, LINCS: a linear constraint solver for molecular simulations, J. Comput. Chem. 18 (1997) 1463-1472.

[34] F. Cao, Y. Liu, J. Xu, Y. He, B. Hammouda, R. Qiao, B. Yang, Probing nanoscale thermal transport in surfactant solutions, Sci. Rep. 5 (2015) 16040.

[35] C. Oldfield, Exchange concept in water-in-oil microemulsions: consequences for 'slow' chemical reactions, J. Chem. Soc. Faraday Trans. 87 (1991) 2607-2612. 
Table 1. The interaction energy of a single water molecule with other molecules when it is located in vacuum, in n-octane fluids at $370 \mathrm{~K}$, and in reverse micelles in microemulsions maintained at $370 \mathrm{~K}$ at different time instants. The interaction energies are computed with different real-space cutoff lengths $\left(r_{c, \text { real }}\right)$ for the electrostatic interactions. The energy is given in unit of $\mathrm{kJ} / \mathrm{mol}$.

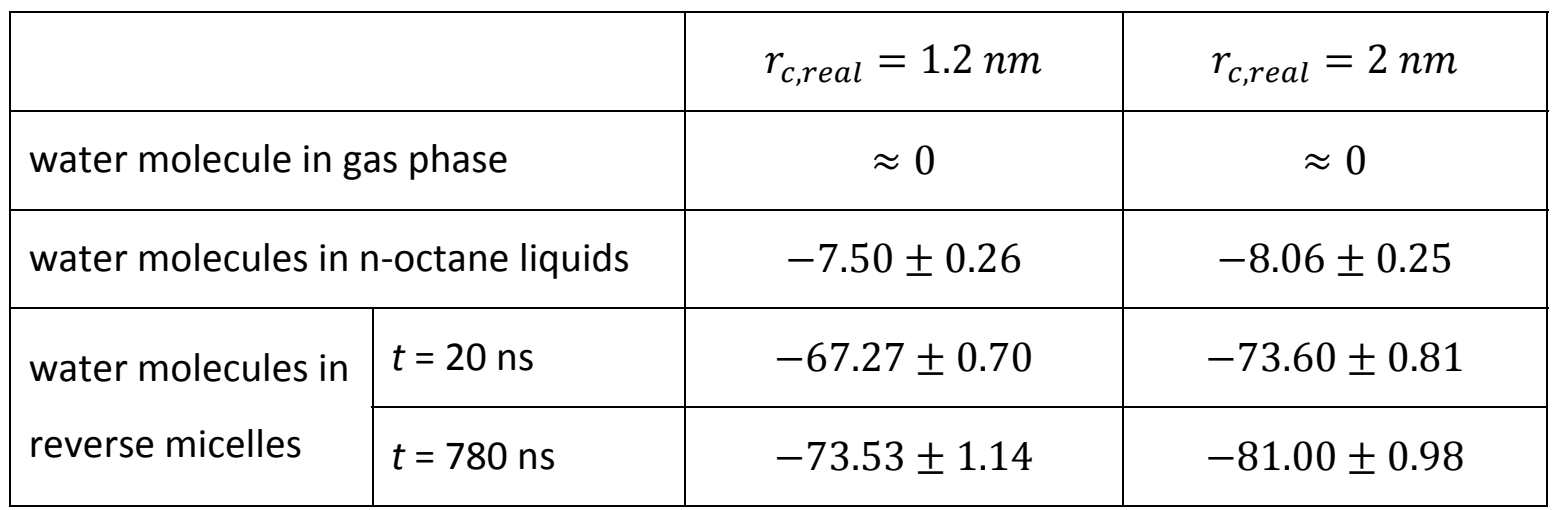




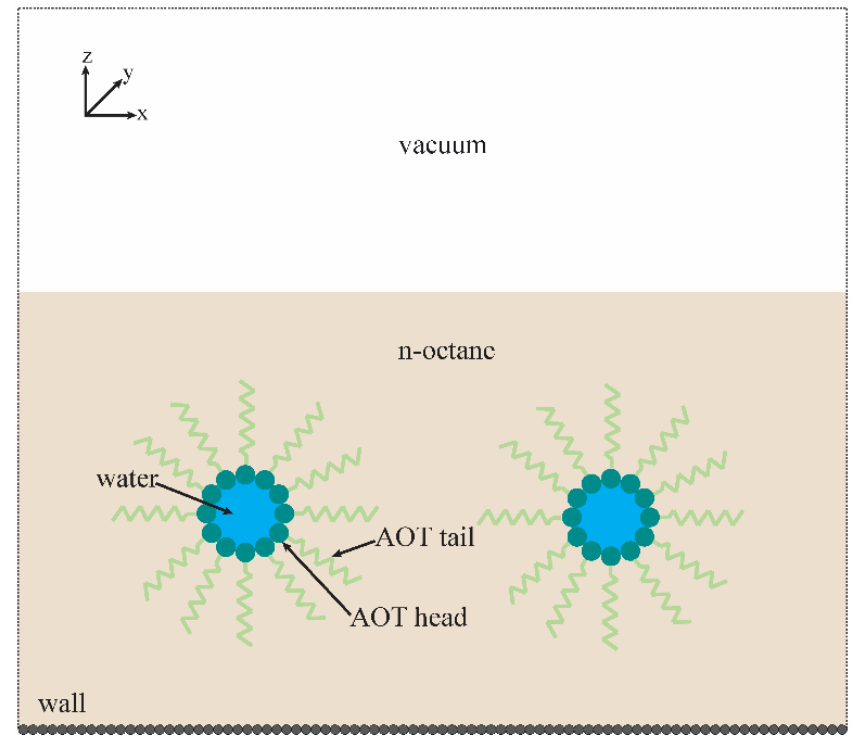

Figure 1. A schematic of the simulation system. The black dashed lines indicate the MD simulation box, which is periodical in all three directions.

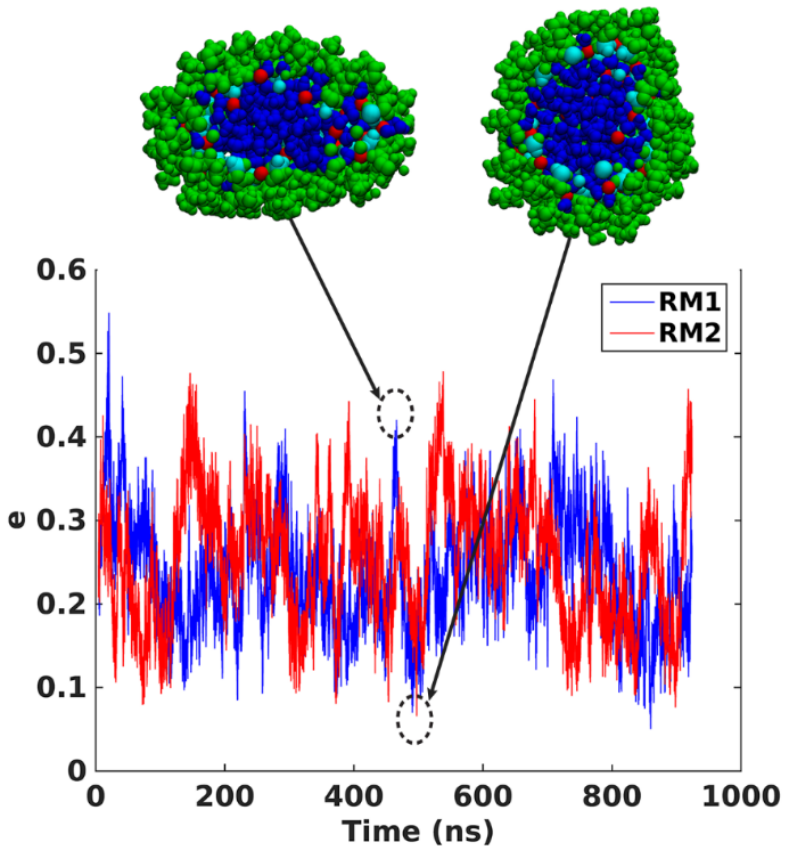

Figure 2. The evolution of the eccentricity of the two AOT reverse micelles in n-octane liquids maintained at $300 \mathrm{~K}$. The insets are cutout-view of the reverse micelles showing that their significant shape fluctuations during the simulation. The cyan, green, and red balls denote the hydrophilic head, hydrophobic tail, and $\mathrm{Na}^{+}$ions of the AOT molecule, respectively. The blue balls denote the water molecules. 


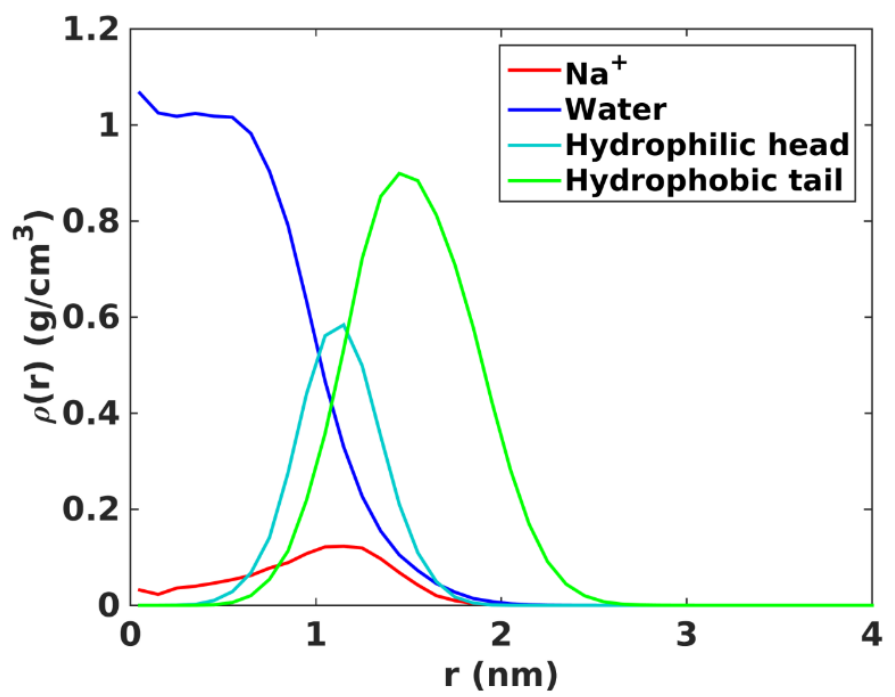

Figure 3. The radial density profiles of the water molecules, the hydrophilic head, the $\mathrm{Na}^{+}$ion, and the hydrophobic tail of the AOT molecules of two stable AOT reverse micelles in n-octane liquids maintained at $300 \mathrm{~K}$. The center-of-mass of the micelle is taken as the origin.

(a)

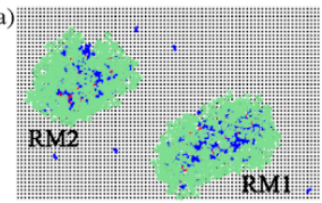

(b)

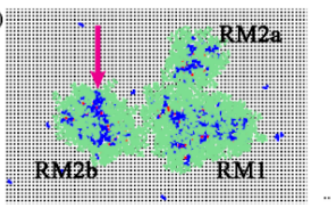

(c)

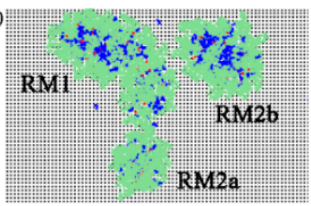

(d)

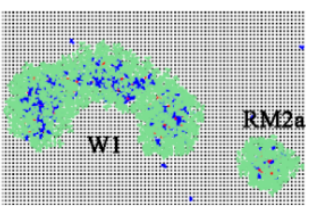

$0 \mathrm{~ns}$

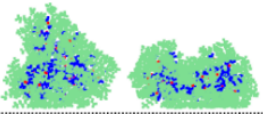

$120 \mathrm{~ns}$
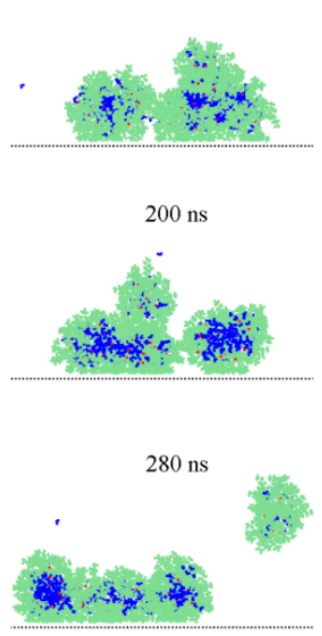

(e)
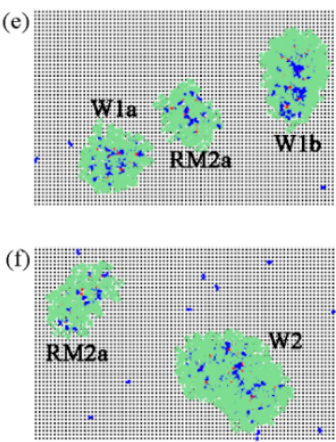

(g)
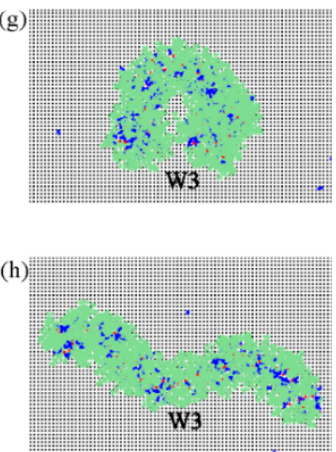

$400 \mathrm{~ns}$

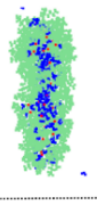

4*

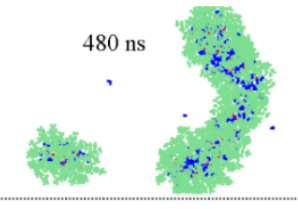

$560 \mathrm{~ns}$

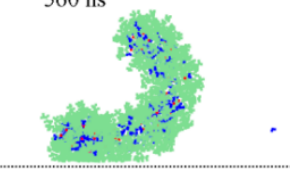

$800 \mathrm{~ns}$

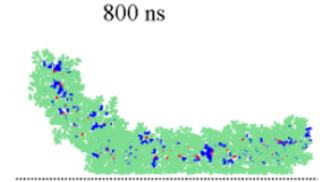

Figure 4. Snapshots of the microemulsion maintained at $370 \mathrm{~K}$ during a $0.8 \mu$ s production run. The green balls denote the AOT molecule (i.e., both its hydrophobic and hydrophilic parts), the red balls denote the $\mathrm{Na}^{+}$ions, the blue balls denote the water molecules, and the black dots denote the wall atoms. The $\mathrm{n}-$ octane molecules are not shown for clarity. For each frame of the snapshots, the left and right panel show the top and side view, respectively. 


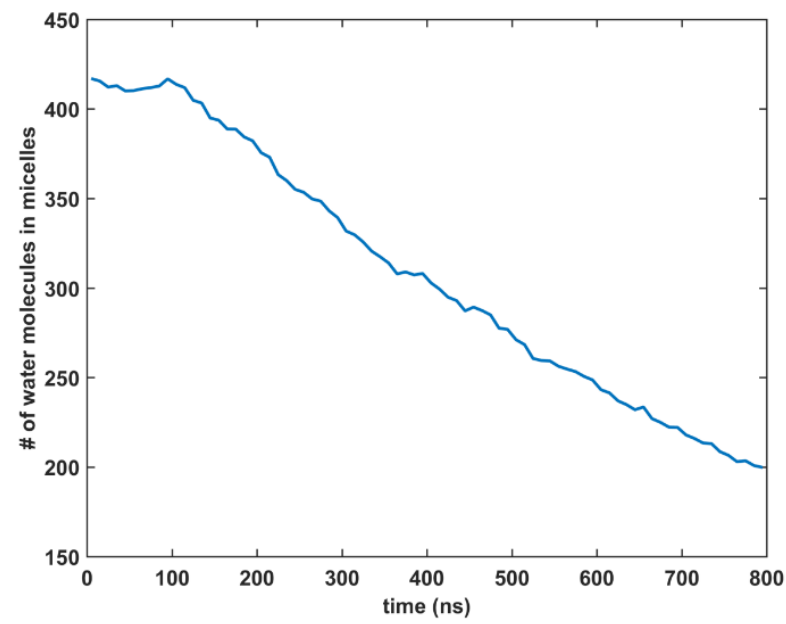

Figure 5. Evolution of the total number of water molecules within the reverse micelles for microemulsions maintained at $370 \mathrm{~K}$.
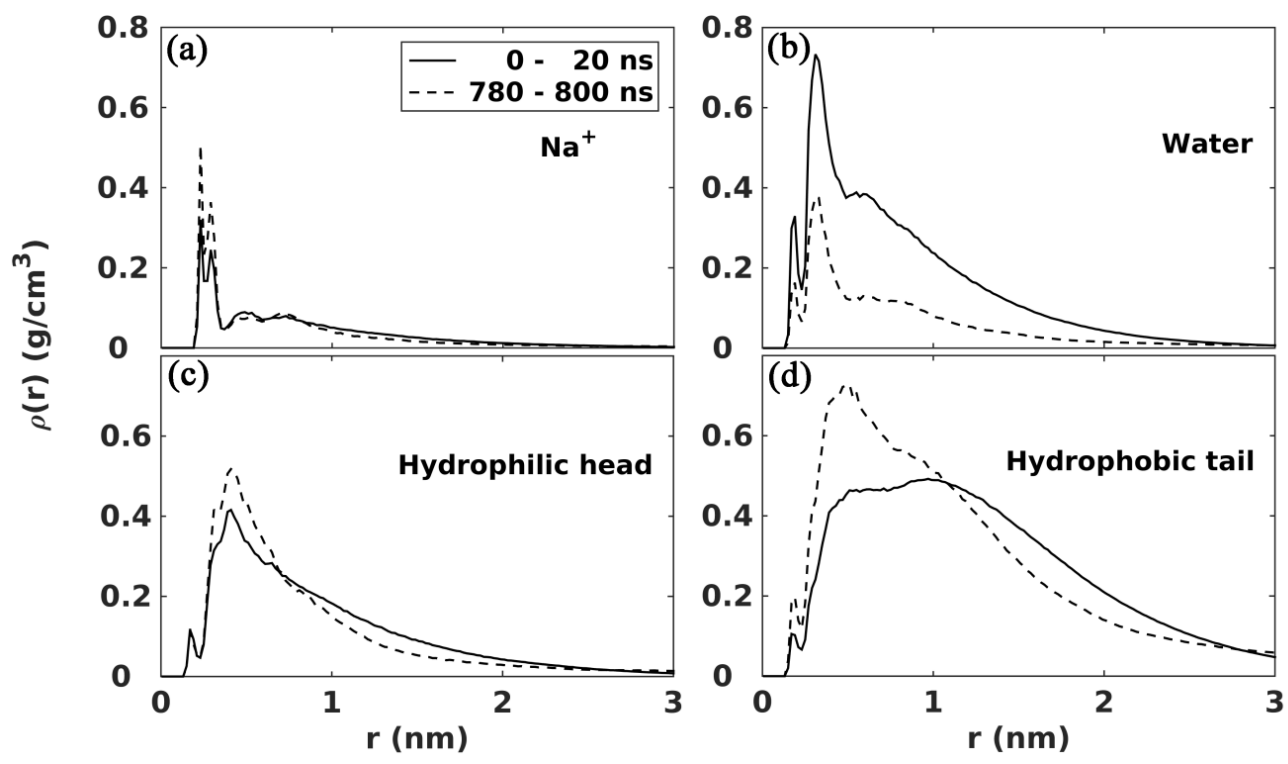

Figure 6. Density distribution profiles of various species around the water molecules within reverse micelles when the microemulsion is maintained at $370 \mathrm{~K}$ at two different time instants (the solid lines denote those for $t=0-20 \mathrm{~ns}$ and the dashed lines denote those for $t=780-800 \mathrm{~ns}$ ). The $\mathrm{Na}^{+}$ions and the AOT's hydrophilic heads are the hydrophilic moieties of the AOT molecule. 


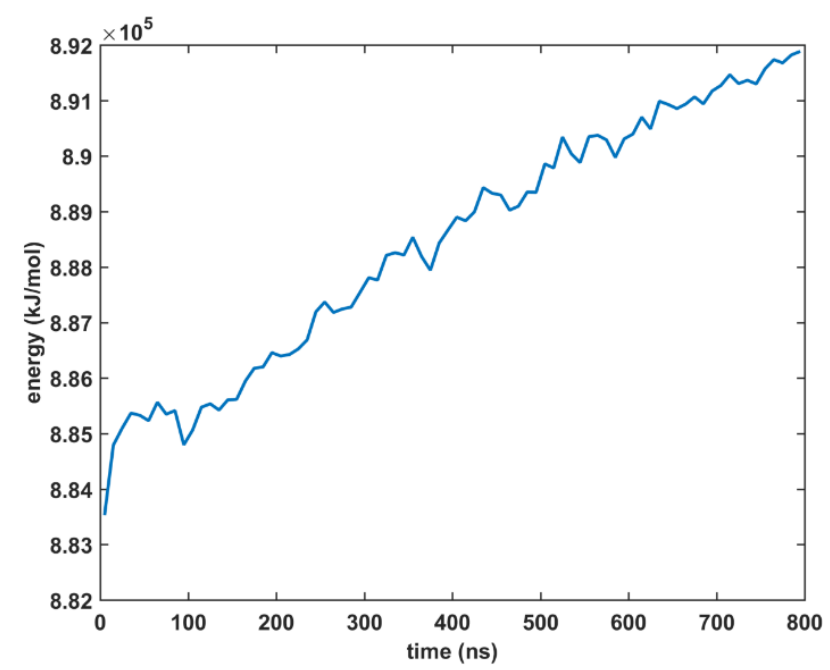

Figure 7. Evolution of the total energy of the microemulsion system maintained at $370 \mathrm{~K}$. 